\title{
Relación entre los hábitos alimentarios y el rendimiento académico en estudiantes de universidades públicas y privadas de la localidad de Chapinero, Bogotá
}

DOI: 10.17533/udea.penh.v23n2a05

PERSPECTIVAS EN NUTRICIÓN HUMANA

ISSN 0124-4108

Escuela de Nutrición y Dietética, Universidad de Antioquia. Medellín, Colombia

Vol. 23, N. ${ }^{\circ}$ 2, julio-diciembre de 2021, pp. 183-195.

Artículo recibido: 22 de septiembre de 2021

Aprobado: 26 de noviembre de 2021

\section{Aleyda Parra-Castillo ${ }^{1 *}$; Liliana Morales-Canedo²; Melisa Medina-Valencia ${ }^{3}$}

\section{-Resumen}

Antecedentes: los hábitos alimentarios inciden directamente en el rendimiento académico. Entre la población de jóvenes universitarios, se ha observado que existe asociación entre los buenos hábitos de alimentación y de estudio, lo que repercute positivamente en su rendimiento académico. Objetivo: determinar la relación existente entre los hábitos alimentarios y el rendimiento académico de estudiantes de universidades públicas y privadas de Chapinero, Bogotá. Materiales y métodos: se aplicó un cuestionario desarrollado por el grupo de investigación GRIINSAN de la Facultad de Nutrición y Dietética de la Universidad del Atlántico entre una muestra de 68 estudiantes de universidades privadas y 53 de universidad pública; los resultados se contrastaron con los registros de desempeño académico de cada uno. Resultados: el consumo diario de azúcares y grasas $(<35 \%)$, la preferencia por alimentos fritos y la no ingesta de media tarde están asociados con un desempeño académico bajo de los estudiantes de universidad pública. El consumo diario de azúcares (26,4\%) y la baja ingesta de verduras y de media tarde están asociados con un desempeño bajo entre los estudiantes de universidad privada. Conclusiones: los estudiantes con desempeño superior muestran hábitos alimentarios más saludables, con mayor consumo de frutas, verduras y media tarde.

Palabras clave: hábitos alimentarios, rendimiento académico, estudiantes, factores socioeconómicos, consumo de alimentos.

1 *Autor de correspondencia. MSc. Docente Universidad del Atlántico, Barranquilla, Colombia. aleydaparra@mail.uniatlantico.edu.co. https://orcid.org/0000-0001-5656-9384

2 Msc. Docente Universidad del Atlántico, Barranquilla, Colombia. lilianamorales@ mail.uniatlantico.edu.co. https://orcid.org/0000-0001-9554-7655

3 Msc. Secretaría de Salud-Subred Integrada de Servicios de Salud-Sur Occidente E.S.E., Bogotá, Colombia. ar.eu.z@hotmail.com. https://orcid.org/0000-0003-4356-5370

Cómo citar este artículo: Parra-Castillo A, Morales-Canedo L, Medina-Valencia M. Relación entre los hábitos alimentarios y el rendimiento académico en estudiantes de universidades públicas y privadas de la localidad de Chapinero, Bogotá. Perspect Nutr Humana. 2021;23:183-95. DOI: 10.17533/udea.penh.v23n2a05 


\section{Relationship Between Eating Habits and Academic Performance in Students of Public and Private Universities in Chapinero, Bogotá}

\section{Abstract}

Background: Eating habits directly affect academic performance. Among young university students, it has been observed that there is an association between good eating and study habits, which has a positive impact on their academic performance. Objective: To determine the relationship between eating habits and academic performance of students from public and private universities in Chapinero, Bogota. Materials and Methods: A questionnaire developed by the GRIINSAN research group of the Faculty of Nutrition and Dietetics of the Universidad del Atlántico was applied among a sample of 68 students from private universities and 53 from public universities; the results were contrasted with the academic performance records of each student. Results: Daily consumption of sugars and fats $(<35 \%)$, preference for fried foods, and the absence of mid-afternoon intake are associated with low academic performance of public university students. The daily consumption of sugars $(26.4 \%)$ and the low intake of vegetables and the mid-afternoon are associated with low performance among private university students. Conclusions: Students with a higher performance show healthier eating habits, with higher consumption of fruits, vegetables, and mid-afternoons.

Keywords: Eating habits, food habits, academic performance, students, socioeconomic factors, food consumption.

\section{INTRODUCCIÓN}

La nutrición comprende todos los procesos fisiológicos que permiten que los organismos puedan aprovechar las sustancias químicas contenidas en los alimentos (1). Debido a su importancia para el bienestar individual y social, es necesario gestionar una nutrición correcta derivada de la ingesta de alimentos saludables que satisfagan los requerimientos mínimos y que, a partir del desarrollo del hábito, se erijan como un factor protector del estado nutricional y de la salud de los individuos (2).

Sin embargo, los hábitos alimenticios saludables no se desarrollan de forma automática, y dependerán en gran medida de las circunstancias socioeconómicas, ambientales y culturales de las personas, así como de las tradiciones familiares que se transmiten de generación en generación $(3,4)$. Tales hábitos pueden ser más o menos saludables, dependiendo de su orientación, y tienen un efecto inevitable sobre la condición general de los individuos en términos físicos y psicológicos.
Diversos estudios empíricos han probado, por ejemplo, que existe una directa correlación entre el estado cognoscitivo y el nutricional, lo que repercute en aspectos cuantificables como el rendimiento académico estudiantil (5-8). De acuerdo con Adelantado-Renau et al. (9), una frecuencia adecuada en el consumo de los alimentos puede contribuir al mejoramiento del rendimiento académico. En tanto autores como Naveed et al. (10) indican que la calidad de los alimentos consumidos también guarda relación directamente proporcional con el rendimiento académico.

Existen, por lo tanto, diferencias consistentes entre el rendimiento académico de individuos de un contexto socioeconómico medio-alto y los de extracciones más bajas. ¿Cómo se dan estas diferencias? ¿Cómo se relacionan los hábitos alimentarios con el desempeño académico de los individuos? ¿Cuáles son los factores prevalentes que tienen incidencia directa al respecto? Para dar respuesta a estos cuestionamientos, se decidió realizar un estudio entre un grupo de estudiantes universitarios con distintos antecedentes, con la 
intención de definir cómo las diferencias en los hábitos alimentarios entre uno y otro grupo incidían en el rendimiento académico de estos (11).

Entender cómo se relacionan los hábitos alimenticios con el rendimiento académico de los estudiantes universitarios dependiendo de su contexto puede contribuir a la gestión de políticas institucionales que respondan de mejor manera a las necesidades específicas de las poblaciones que se atienden. En ese sentido, que las universidades conozcan cómo el aspecto nutricional incide en el desempeño académico de sus estudiantes puede guiar la adopción de medidas que no solo favorezcan el rendimiento de los individuos, sino que además repercutan en su estado de salud, minimizando el riesgo de que desarrollen enfermedades crónicas no transmisibles como la hipertensión arterial, la diabetes mellitus tipo 2, dislipidemias, enfermedades cardiovasculares, alteraciones del estado nutricional, sobrepeso/ obesidad, entre otras $(12,13)$.

Establecer este análisis a partir del rendimiento académico implica entender que el desempeño de un estudiante depende de diferentes factores, entre los que se cuentan los nutricionales (14). A este subyacen aspectos como la disponibilidad, el acceso y el tipo de alimentos consumidos, así como la frecuencia de ingesta. Los resultados derivados permitirán comprender mejor el fenómeno de la nutrición universitaria y constituirán un insumo importante para identificar los factores de mayor relevancia en la correlación del desempeño académico con los hábitos alimentarios, de manera que se puedan establecer políticas tendientes a la mejora, tanto de los indicadores académicos como de los nutricionales. En tal sentido, el objetivo del estudio fue determinar la relación existente entre los hábitos alimentarios y el rendimiento académico en estudiantes de uni- versidades públicas y privadas de la localidad de Chapinero, Bogotá.

\section{MATERIALES Y MÉTODOS}

Se realizó un estudio de tipo descriptivo correlacional de corte transversal (15). La población de estudio estuvo conformada por 248 estudiantes en cuarto semestre de Ingeniería de Sistemas (a corte del año 2019) de las instituciones privadas Universidad Católica de Colombia y Universidad Piloto de Colombia, y por los 115 estudiantes en cuarto semestre de Ingeniería de Sistemas (a corte del año 2019) de la institución pública Universidad Distrital Francisco José de Caldas, todas con ubicación geográfica en la localidad de Chapinero, Bogotá. Los criterios para la caracterización de la población obedecen a la necesidad de establecer juicios unificados que permitan una valoración académica equiparable. No es posible establecer un contraste verosímil entre estudiantes de carreras diferentes y en semestres distintos, toda vez que cada carrera tiene sus propios y particulares niveles de dificultad, los cuales varían en función del grado de profundización en que se esté. Por ello, para obtener una evaluación equitativa, se optó por solo contemplar las poblaciones de estudiantes de Ingeniería de Sistemas de cuarto semestre de las universidades en cuestión.

Se estableció un muestreo probabilístico, con el fin de garantizar la misma posibilidad de participación para cada individuo; se escogieron aleatoriamente (16). Las muestras definidas para un nivel de confianza del $95 \%$ y un margen de error del $10 \%$ fueron de 53 individuos de universidad pública y 68 individuos de universidades privadas. Entre los criterios de inclusión se tiene que los participantes fueran mayores de edad, estudiantes activos (matriculados académica y financieramente en las universidades objeto de estudio) y que manifestaran de forma expresa y voluntaria — previa 
información - su consentimiento para hacer parte del estudio.

Se partió de la hipótesis nula de que no existe asociación entre las variables categóricas enunciadas, y se definió el nivel de confianza en el orden del $95 \%$. Medidas de las pruebas que arrojen valores de $p$ menores al $5 \%$ invitan a rechazar la hipótesis nula y a concluir que sí existe asociación entre las variables estudiadas, en tanto que medidas superiores al $5 \%$ dan cuenta de lo contrario.

Entre los integrantes de las muestras definidas, se aplicó un cuestionario de hábitos alimentarios diseñado y validado en el seno del grupo de investigación GRIINSAN de la Facultad de Nutrición y Dietética de la Universidad del Atlántico, a partir del criterio de expertos y del índice de consistencia interna que provee el alfa de Cronbach, que para el caso tuvo un valor válido de 0,9. El cuestionario compila 26 preguntas destinadas a conocer las características sociodemográficas (edad, sexo y estrato), la disponibilidad y el acceso a los alimentos, sus hábitos y frecuencias de consumo (ingesta de frutas, verduras, vegetales, grasas, etc.). El detalle de las preguntas formuladas a los participantes en el cuestionario se relaciona en el anexo. Los datos derivados se contrastaron con el último informe del promedio académico de los estudiantes que participaron en la investigación, provisto de forma libre por estos bajo la garantía de que su identidad fuera siempre preservada.

En cuanto a las variables de interés, el desempeño académico se ajustó al sistema de calificación colombiano, que va de uno a cinco y que considera aprobado al individuo con una nota igual o mayor a tres. En este caso, el desempeño académico fue clasificado como bajo cuando el promedio de las notas estaba entre 3 y 3,5 ; medio cuando era mayor a 3,5 y menor o igual a 4; y alto cuando se hallaba entre 4,1 y 5 . Para ello, se sumaron las notas obtenidas en cada una de las asignaturas cursadas y el total obtenido se dividió entre el total de asignaturas vistas durante el semestre inmediatamente anterior a la aplicación del cuestionario de hábitos nutricionales.

En relación con el estrato socioeconómico, este es una medida estandarizada en Colombia establecida de acuerdo con la ubicación de la vivienda y de su entorno, que contempla seis estratos, de los cuales el 1 corresponde al más bajo y 6 al más alto (17). Por otra parte, los datos para la frecuencia de consumo de los alimentos y los tipos de alimentos consumidos se recolectaron a partir del cuestionario de hábitos nutricionales.

\section{Análisis estadístico}

La tabulación y análisis de la información se realizó a través del programa SPSS Statistics 25. Primero, se compararon las características sociodemográficas de las muestras para determinar la semejanza de estas a partir de la prueba de proporciones para muestras independientes $Z$, con un nivel de confianza del $95 \%$. Como medida de asociación entre las variables categóricas hábitos alimentarios (conformados por subvariables como la frecuencia de consumo y los tipos de alimentos) y desempeño académico (bajo, medio y alto) se emplearon las pruebas de ji al cuadrado para analizar las correspondencias con frecuencias esperadas que no presentaran datos inferiores a cinco en una proporción igual o mayor al $20 \%$ y y la prueba exacta de Fisher para los casos contrarios.

\section{Consideraciones éticas}

El proceso de estudio se ciñó a los principios éticos establecidos en la declaración de Helsinki para las investigaciones médicas en seres humanos de la Asociación Médica Mundial (AMM) (18). De la legislación nacional se tomaron en consideración las regulaciones expuestas en la Resolución 
8430 de 1993, a través de la cual se establecen las normas científicas, técnicas y administrativas que deben considerarse para el desarrollo de investigaciones en salud. Asimismo, a lo largo del estudio - categorizado de riesgo mínimo, según los lineamientos del Ministerio de Salud (19)_, se preservaron los derechos, la dignidad, los intereses y la sensibilidad de las personas involucradas, garantizando la absoluta confidencialidad de los datos derivados. El Comité Misional del programa de Nutrición y Dietética de la Universidad del Atlántico fue el encargado de la supervisión del desarrollo investigativo en su aspecto ético. Los participantes fueron informados previamente sobre las implicaciones del estudio y se les consultó si estaban dispuestos a participar en él bajo la garantía absoluta del anonimato y de que los datos no serían compartidos con terceros.

\section{RESULTADOS}

Los datos sociodemográficos de los participantes se comparten en la tabla 1. Se realizó la comparación de los datos de una muestra y otra para corroborar la semejanza de ambos grupos a partir de la prueba de proporciones $Z$. Se encontró que para los aspectos clave como edad y sexo existía semejanza entre las medias de ambas muestras, por lo que las comparaciones subsiguientes se consideraron válidas.

En adelante, la caracterización de los hábitos alimenticios a partir de la aplicación del cuestionario empleado para tal fin arrojó que el desayuno era la única comida de cobertura global, en tanto que el consumo de refrigerios en media mañana y en media tarde tenía mayor prevalencia entre los estudiantes de universidades privadas.

Tabla 1. Factores sociodemográficos y rendimiento académico de los estudiantes según tipo de universidad

\begin{tabular}{|c|c|c|c|c|c|c|}
\hline \multirow{3}{*}{ Variable } & \multirow{3}{*}{ Estratificación } & \multicolumn{4}{|c|}{ Tipo de universidad } & \multirow{3}{*}{ Valor de $\mathrm{p}^{*}$} \\
\hline & & \multicolumn{2}{|c|}{ Pública (n=53) } & \multicolumn{2}{|c|}{ Privada $(n=68)$} & \\
\hline & & Frec & $\%$ & Frec & $\%$ & \\
\hline \multirow{2}{*}{ Sexo } & Hombres & 37 & 69,8 & 44 & 64,7 & 0,554 \\
\hline & Mujeres & 16 & 30,1 & 24 & 35,2 & 0,553 \\
\hline \multirow{3}{*}{ Grupo de edad } & $18-20$ años & 25 & 47,1 & 38 & 55,8 & 0,341 \\
\hline & 21-24 años & 14 & 26,4 & 20 & 29,4 & 0,715 \\
\hline & $\geq 25$ & 14 & 26,4 & 10 & 14,7 & 0,109 \\
\hline \multirow{6}{*}{ Estrato socioeconómico } & 1 & 5 & 9,4 & 0 & 0,0 & \multirow{6}{*}{ No aplica } \\
\hline & 2 & 10 & 18,8 & 10 & 14,7 & \\
\hline & 3 & 11 & 20,7 & 10 & 14,7 & \\
\hline & 4 & 27 & 50,9 & 30 & 44,1 & \\
\hline & 5 & 0 & 0,0 & 18 & 26,47 & \\
\hline & 6 & 0 & 0,0 & 0 & 0,0 & \\
\hline \multirow{3}{*}{ Desempeño académico } & Bajo & 21 & 39,6 & 24 & 35,2 & 0,619 \\
\hline & Medio & 12 & 22,6 & 27 & 39,7 & 0,045 \\
\hline & Alto & 20 & 37,7 & 17 & 25 & 0,132 \\
\hline
\end{tabular}

* Valor de p según la prueba de proporciones Z. 
El consumo de almuerzo y cena era prácticamente generalizado $(95,5 \%$ en universidad privada y $96,2 \%$ en universidad pública), y no se identificó injerencia significativa del consumo de carnes, leguminosas, cereales, lácteos, raíces y comidas rápidas sobre el rendimiento de los estudiantes ( $p$-valores derivados de la prueba ji al cuadrado 0 Fisher superiores a 0,05 ).

En la tabla 2 se comparte el resumen de resultados y los valores de probabilidad que se asocian con los aspectos evaluados y que se consideran relevantes. De acuerdo con los datos analizados, los hábitos de consumo vinculados al tipo de alimentos y a la costumbre de tomar refrigerios incide de alguna manera en el desempeño de los estudiantes (existen algunas distinciones que dependen de la procedencia académica).

En cuanto a la frecuencia de consumo y de los niveles de rendimiento asociados a esta en relación con las variables caracterizadas, se identificó que existen diferencias puntuales entre el rendimiento académico de estudiantes de universidades privadas y universidad pública, en tanto los estudiantes de instituciones privadas muestran un mejor rendimiento si el número de personas para el que se cocina es mayor. Lo contrario sucede con los estudiantes de universidad pública, quienes tienen un mejor rendimiento si el número de personas para el que se cocina es menor. Algo similar pasa con el consumo de media mañana, que no incide de forma significativa en la mejora del desempeño de los estudiantes de universidades privadas, pero sí lo hace en el desempeño de los estudiantes de universidad pública de acuerdo con la información recolectada. Por su parte, se observó que el consumo de media tarde incide positivamente en el rendimiento académico de ambos grupos de participantes, siendo menor el desempeño de quienes no la consumen. Esto se corresponde directamente con lo expresado por la mayoría de los participantes que indicaron que solían sentir más hambre por la tarde.

Ahora bien, en lo que tiene que ver con la frecuencia de consumo de frutas y verduras, se logró establecer que el rendimiento académico es superior en la medida en que su consumo es más frecuente. El referido dato aplica para ambos grupos, mostrándose un mejor desempeño en quienes las consumen diariamente. Por el contrario, la ingesta de azúcares va en detrimento del rendimiento académico de los participantes de ambos grupos en tanto más asidua sea.

Con la predilección por los alimentos fritos se observa una ambigüedad: mientras que la preferencia por las frituras demuestra resentir el desempeño académico de los estudiantes de universidad pública, no tiene ninguna incidencia significativa sobre el rendimiento de los estudiantes de universidades privadas. Con las grasas, en tanto, no se halló una relación significativa en ninguno de los dos grupos analizados.

En cuanto al rendimiento académico, tal como se aprecia en la parte inferior de la tabla 1, no se identificaron diferencias significativas entre los estudiantes de un grupo y otro en los rangos alto y bajo. Por otra parte, se encontraron diferencias significativas en las proporciones de estudiantes con calificación media entre grupos, siendo mayor la citada proporción en la universidad privada.

\section{DISCUSIÓN}

Los resultados compartidos permiten identificar que existen diferencias mínimas entre los hábitos alimentarios de estudiantes de universidades privadas y los hábitos alimentarios de estudiantes de universidad pública. 


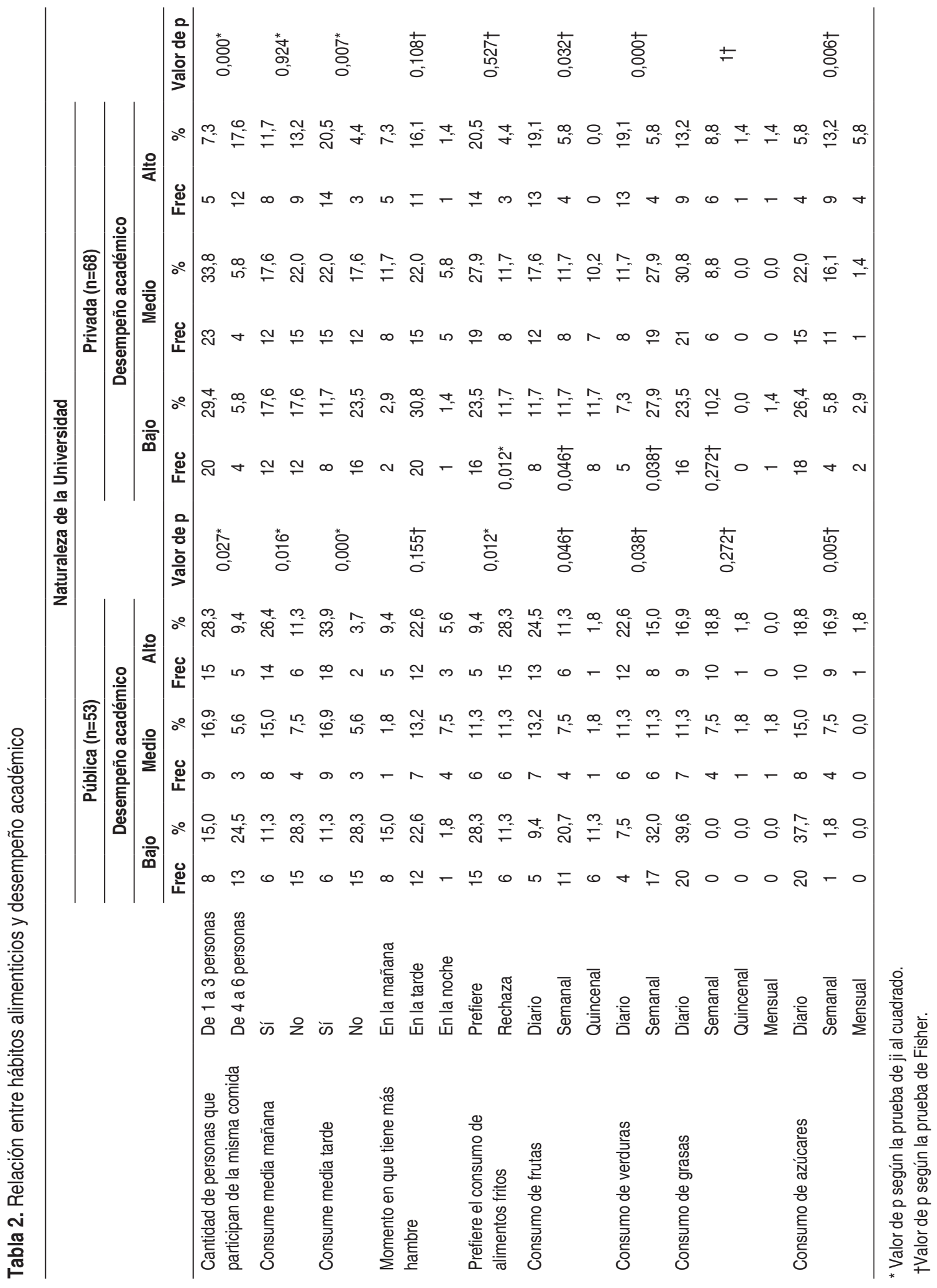


Esta situación puede explicarse con base en las tendencias alimenticias de los bogotanos en general, entre los cuales prevalece el consumo de bebidas azucaradas, grasa saturada, golosinas y dulces, y un bajo consumo de cereales integrales, frutas y lácteos (20). Asimismo, las coincidencias pueden ser explicadas por las semejanzas socioeconómicas identificadas (recuérdese que en ambos grupos caracterizados los estratos socioeconómicos prevalentes fueron el 3 y el 4), así como por el tamaño de la muestra y la delimitación seleccionada, lo que implicó que los estudiantes estuvieran adscritos a un único programa y semestre.

Las semejanzas persisten cuando se analiza la relación entre tales hábitos y el rendimiento académico de los estudiantes seleccionados como muestra representativa para ambas poblaciones. En los dos casos, aspectos como el número de individuos que se alimentan de una misma preparación, el consumo de refrigerio de media mañana y de media tarde, la predilección por alimentos fritos y el consumo de frutas, verduras y azúcares inciden de forma significativa en el rendimiento académico estudiantil. Estos resultados se ajustan a lo sustentado por Correa-Burrows et al. (21), quienes lograron demostrar que los hábitos alimenticios constituyen un factor incidente en el desempeño académico de los estudiantes, siendo este mayor cuando los reseñados hábitos son saludables y menor cuando no lo son.

Sin embargo, al valorar independientemente cada una de las subvariables referenciadas, se identificaron las primeras diferencias puntuales. Por ejemplo, en lo que corresponde al número de individuos que se alimentan de una misma preparación, se observa que, en la medida en que la cantidad de personas aumenta, el rendimiento de los estudiantes de universidades privadas mejora y el de los estudiantes de universidad pública des- mejora. La explicación a este fenómeno puede asociarse a las condiciones económicas de uno y otro grupo, como lo sostienen Torres-Cárdenas et al. (22) y Gil-Gil et al. (23). Mientras que en familias que pertenecen a estratos socioeconómicos más altos el aumento en la cantidad de comida que se requiere para atender un mayor número de individuos puede ser suplido de forma eficiente, es usual que, a menor nivel de ingresos, aspectos como la calidad y la cantidad de los alimentos disminuya para poder hacer reparticiones equitativas. Este análisis sugiere que una buena prospectiva para el estudio desarrollado es tomar como medida de asociación el estrato socioeconómico, por lo que sería pertinente realizar un análisis estratificado de la población de interés.

Algo similar sucede con el consumo de media mañana. Mientras que en la muestra correspondiente a estudiantes de universidades privadas se observó un comportamiento semejante en el rendimiento académico, independientemente de si ingerían un refrigerio o no en ese espacio de tiempo, en el caso de los estudiantes de universidad pública se observó un rendimiento académico mayormente desfavorable entre los estudiantes que no merendaban a media mañana. En este caso, como bien lo explican Gil-Gil et al. (23), las demandas y deficiencias calóricas tienen una función determinante, pues los casos de malnutrición suelen ser mucho más comunes entre la población con menores recursos, siendo la desnutrición la principal consecuencia del fenómeno entre los individuos con menores recursos y la sobrenutrición la consecuencia prevalente entre los de mayores ingresos.

Por su parte, la ingesta de refrigerio de media tarde repercutía favorablemente en ambos grupos de manera clara, lo que se justifica con la aseveración común de que la tarde era el momento en que más hambre padecían los encuestados. 
Al respecto, es pertinente indicar que en ambos casos se identificó prevalencia de estudiantes que no consumían media tarde, y se observó en estos casos mayor presencia de rendimientos académicos bajo y medio. Al respecto, Ibarra et al. (24) indican que el consumo de una comida ligera en la tarde repercute positivamente en el rendimiento académico de los estudiantes. García (25) sostiene que la racionalización de las calorías ingeridas favorece el sistema nervioso central, lo que, aunado a otros aspectos, contribuye a una mejor concentración y, en consecuencia, a un mejor rendimiento académico.

En lo que tiene que ver con el consumo de frutas, verduras y azúcares, se observan tendencias de desempeño académico semejantes en la medida en que a mayor frecuencia de consumo de frutas y verduras existe un mejor rendimiento académico en ambos casos, y que a mayor frecuencia de consumo de azúcares existe una desmejora perceptible en el promedio de calificaciones de los participantes (indistintamente de su procedencia académica). Esta valoración responde de manera precisa a lo planteado por López-Nuevo et al. (11), quienes sostienen que los hábitos alimentarios poco saludables pueden determinar un desempeño académico negativo, mientras que los hábitos saludables pueden generar el resultado opuesto.

Ya se reseñó la ambigüedad identificada en torno a una de las restantes subvariables: la predilección por alimentos fritos. Mientras que para los estudiantes de universidades públicas la aceptación y asiduidad de consumo deriva en peores calificaciones, en los estudiantes de universidades privadas el efecto no es significativo. A ese respecto, sería prudente ahondar - a modo de prospectiva - en los hábitos particulares de los individuos en tal sentido, esto es, tipo de fritura, tipo de aceite empleado y lugar de ingesta (26). El consumo de grasas no demostró incidir significativamente en ninguno de los grupos, lo que merece ser analizado en sucesivas investigaciones a partir de la valoración nutricional completa de los individuos, incluyendo aspectos como la talla y el Índice de Masa Corporal (IMC), como lo sugieren los estudios de Peralta et al. (27) y de Landeros et al. (28).

Al contrastar de forma generalizada los resultados, se tiene que el rendimiento académico de los estudiantes de universidad pública es ligeramente superior al de los estudiantes de universidades privadas en el rango alto (aunque la citada diferencia no puede anotarse como significativa). Las proporciones de estudiantes en nivel medio y bajo es mejor en las universidades privadas, cuyas diferencias solo son significativas en la valoración que implica un promedio superior a 3,5 y menor o igual 4,5. En todo caso, el análisis de los datos arroja decididamente que los individuos con desempeño alto (tanto en instituciones oficiales como no oficiales) suelen mostrar hábitos alimentarios más sanos: mayor consumo de frutas y verduras, ingesta de media tarde y menor consumo de azúcares y de grasas (esto último, en el caso de los estudiantes de institución pública). Factores como la edad y el sexo no tuvieron mayor injerencia sobre los resultados, pues no se identificaron diferencias puntuales en tal sentido.

Los datos derivados del presente estudio permiten confirmar algunos aspectos relevantes en relación con los hábitos alimentarios y el rendimiento académico universitario. Por ejemplo, es claro que mejores costumbres en el consumo de alimentos favorecen el desempeño, pues los procesos cognoscitivos dependen en gran medida del grado de nutrición que se posea (29). Del mismo modo, aspectos como la actitud, la atención y la disposición están íntimamente relacionados con la calidad de la nutrición a la que tenga la posibili- 
dad de acceder cada persona; la influencia es de doble vía, como lo sostienen Trigueros et al. (30). En ese orden de ideas, como lo sugieren TorresCárdenas et al. (22) y Gil-Gil et al. (23), aunque es lógico que el nivel de ingresos que posea cada individuo favorezca el proceso, debe reconocerse que no es el único inductor. En efecto, como pudo comprobarse al contrastar la cantidad de participantes con rendimientos académicos bajo, medio y alto, en la educación nutricional inciden decididamente los hábitos nutricionales que cada individuo posea.

Si bien el rendimiento académico no puede ser asociado únicamente con los hábitos alimentarios como variables que lo determinan (factores institucionales, pedagógicos, psicosociales y sociodemográficos tienen también enorme relevancia al respecto), se ha comprobado que las costumbres inciden en la nutrición de los estudiantes y, por tanto, en el desempeño académico de estos.

En la selección de las muestras no se hallaron diferencias significativas entre los estratos socioeconómicos de los estudiantes de universidades públicas y privadas de Chapinero, lo que no permitió correlacionar esta variable con los otros aspectos evaluados. En ciertos contextos, se esperaría que, en relación con sus hábitos alimentarios, el rendimiento de los individuos que pertenecen a instituciones privadas fuera superior al manifestado por los estudiantes que pertenecen a instituciones oficiales. Sin embargo, la evidencia derivada del presente estudio demuestra que las costumbres nutricionales, si bien dependen de las posibilidades económicas hasta cierto punto, necesitan ser definidas a partir de la educación. Esta, a su vez, está determinada por las tradiciones familiares (31) y las dinámicas sociales $(4,32,33)$, de manera que, indistintamente de los escenarios socioeconómicos, es posible identificar cierta homogeneidad en los hábitos alimentarios.

Como conclusión generalizada del análisis, se tiene que en las universidades públicas existe una mayor proporción de estudiantes con rendimiento alto; sin embargo, es evidente un mejor desempeño estudiantil en universidades privadas al evaluar las proporciones de estudiantes con rendimiento medio y bajo. Tales diferencias pueden ser atribuidas, en buena medida, a los hábitos alimentarios de cada grupo, lo que compagina con lo propuesto por Adelantado-Renau et al. (9), quienes sostienen que aspectos como las frecuencias de consumo y las características de los alimentos consumidos son variables incidentes en el rendimiento académico de los estudiantes.

En relación con el objetivo del estudio, se pudo concluir que los hábitos alimentarios inciden de forma directa en los estudiantes universitarios, independiente de sus antecedentes socioeconómicos. En todo caso, existen diferencias puntuales en relación con las circunstancias y los hábitos que determinan dicho efecto en uno u otro grupo, razón por la cual es necesario que se realicen análisis particulares en cada escenario para proponer políticas que atiendan a las necesidades caracterizadas.

A partir de los resultados de la investigación, se invita a las instituciones universitarias - sin importar su carácter- a promover espacios de sensibilización. Asimismo, pueden proponerse programas para la entrega de meriendas de media tarde entre los estudiantes de universidades (especialmente públicas) con el objetivo de suplir sus carencias al respecto. Esto no solo contribuye a una mejor nutrición estudiantil, sino que favorece el desempeño académico y la valoración institucional. Como prospectiva investigativa, es pertinente valorar la relación entre las variables de interés 
ampliando el rango poblacional, de manera que se tenga una visión mucho más amplia del fenómeno en entornos con menores semejanzas contextuales. Asimismo, se recomienda establecer análisis semejantes que relacionen las variables abordadas directamente con el estrato socioeconómico e incluir valoraciones que integren aspectos como la talla y el IMC de los estudiantes.

En conclusión, independiente de la procedencia de los estudiantes universitarios, se encontró que los hábitos alimentarios inciden directamente en el desempeño académico de estos; su rendimiento es mayor en la medida en que más saludables sean sus hábitos de alimentación (mayor consumo de frutas, verduras y refrigerios de media mañana y media tarde; menor consumo de azúcares y grasas).

\section{AGRADECIMIENTOS}

Al grupo de investigación GRIINSAN de la Facultad de Nutrición y Dietética de la Universidad del Atlántico, a los estudiantes de la Universidad Católica de Colombia, de la Universidad Piloto de Colombia y de la Universidad Distrital Francisco José de Caldas del programa de Ingeniería de Sistemas, quienes participaron de forma voluntaria en el estudio y estuvieron dispuestos a compartir información valiosa para su desarrollo.

\section{CONFLICTO DE INTERESES}

No existe conflicto de interés alguno, pues el estudio fue realizado con recursos propios y no está influido por presiones externas.

\section{Referencias}

1. Cederholm T, Barazzoni R, Austin P, Ballmer P, Biolo G, Bischoff SC, et al. ESPEN guidelines on definitions and terminology of clinical nutrition. Clin Nutr. 2017;36(1):49-64. https://doi.org/10.1016/j.clnu.2016.09.004

2. Popkin BM, Corvalan C, Grummer-Strawn LM. Dynamics of the double burden of malnutrition and the changing nutrition reality. Lancet. 2020;395(10217):65-74. https://doi.org/10.1016/s0140-6736(19)32497-3

3. Molina P, Gálvez P, Stecher MJ, Vizcarra M, Coloma MJ, Schwingel A. Family influences on maternal feeding practices of preschool children from vulnerable families in the Metropolitan Region of Chile. Aten Primaria. 2021 Nov 1;53(9). https://doi.org/10.1016/j.aprim.2021.102122

4. Farrow C. A comparison between the feeding practices of parents and grandparents. Eat Behav. 2014;15(3):339-42. https://doi.org/10.1016/j.eatbeh.2014.04.006

5. Prado EL, Dewey KG. Nutrition and brain development in early life. Nutr Rev. 2014;72(4):267-84. https://doi.org/10.1111/nure.12102

6. López-Jaramillo P, Otero J, Camacho PA, Baldeón M, Fornasini M, López-Jaramillo $\mathrm{P}$, et al. Reevaluating nutrition as a risk factor for cardio-metabolic diseases. Colomb Med. 2018;49(2):175-81. https://doi.org/10.25100/cm.v49i2.3840

7. Giri SP, Biswas S, Bose K. Head Circumference based nutritional status of Rural Bengalee preschool children from Sagar Island, West Bengal, India. Mankind Q. 2018;58:4-599. https://doi.org/10.46469/mq.2018.58.4.5

8. Sibbald CA, Nicholas JL, Chapnick M, Ross N, Gandor PL, Waters WF, et al. Fetal brain ultrasound measures and maternal nutrition: A feasibility study in Ecuador. Am J Hum Biol. 202133(2):e23467. https://doi.org/10.1002/ajhb.23467

9. Adelantado-Renau M, Jiménez-Pavón D, Beltran-Valls MR, Moliner-Urdiales D. Independent and combined influence of healthy lifestyle factors on academic performance in adolescents: DADOS Study. Pediatr Res. 2019;85(4):456-62. https://doi.org/10.1038/s41390-019-0285-z 


\section{Hábitos alimentarios y rendimiento académico en universitarios}

10. Naveed S, Lakka T, Haapala EA. An overview on the associations between health behaviors and brain health in children and adolescents with special reference to diet quality. Int J Environ Res Public Health. 2020 Feb 4;17(3):953. https://doi.org/10.3390/ijerph17030953

11. López-Nuevo CE, Molina JS, Ureña GD. Adherence to healthy habits and academic performance in Vocational Education Students. Retos. 2021;42:118-25. https://doi.org/10.47197/retos.v42i0.87138

12. Wells JC, Sawaya AL, Wibaek R, Mwangome M, Poullas MS, Yajnik CS, et al. The double burden of malnutrition: Aetiological pathways and consequences for health. Lancet. 2020 Jan 4;395(10217):75-88. https://doi.org/10.1016/S0140-6736(19)32472-9

13. Ghattas H, Jamaluddine Z, Akik C. Double burden of malnutrition in children and adolescents in the Arab region. Lancet Child Adolesc Heal. 2021;5(7):462-4. https://doi.org/10.1016/S2352-4642(21)00162-0

14. Chong EG. Factores que inciden en el rendimiento académico de los estudiantes de la Universidad Politécnica del Valle de Toluca. Revista Latinoamericana de Estudios Educativos (México). 2017;XLVII(1): 91-108. https://doi.org/10.37117/s.v1i4.34

15. Gallardo EE. Metodología de la Investigación. Huancayo-Perú: Universidad Continental; 2017, 96 pp. Disponible en: https://repositorio.continental.edu.pe/bitstream/20.500.12394/4278/1/DO_UC_EG_MAI_UC0584_2018.pdf

16. Otzen T, Manterola C. Técnicas de Muestreo sobre una Población a Estudio Sampling Techniques on a Population Study. Int J Morphol. 2017;35(1):227-32. https://doi.org/10.4067/S0717-95022017000100037

17. Colombia. Departamento Nacional de Planeación. Evaluación de la estratificación socio-económica. Bogotá; 2008. [Citado marzo de 2020]. Disponible en: https://www.dnp.gov.co/Programas/Sinergia/EvaluacionesEstratégicas/Evaluacionesdelm pacto/ EstratificaciónSocioEconómica.aspx

18. Declaración de Helsinki de la AMM - Principios éticos para las investigaciones médicas en seres humanos - WMA - The World Medical Association [Internet]. [Citado julio de 2021]. Disponible en: https://www.wma.net/es/policies-post/declaracionde-helsinki-de-la-amm-principios-eticos-para-las-investigaciones-medicas-en-seres-humanos/

19. Lopera MM. Revisión comentada de la legislación colombiana en ética de la investigación en salud. Biomédica. 2017;37(4):577-89. https://doi.org/10.7705/biomedica.v37i4.3333

20. Fajardo E, Camargo Y, Buitrago E, Peña L, Rodríguez L. Estado nutricional y preferencias alimentarias de una población de estudiantes universitarios en Bogotá. Rev Med. 2016;24(2):58-65. https://doi.org/10.18359/rmed.2641

21. Correa-Burrows P, Burrows R, Blanco E, Reyes M, Gahagan S. Nutritional quality of diet and academic performance in Chilean students. Bull World Health Organ. 2016;94(3):185-92. https://doi.org/10.2471/BLT.15.161315

22. Torres-Cárdenas M, Pérez B, Landaeta-Jiménez M, Vásquez-Ramírez M. Consumo de alimentos y estado nutricional según estrato socioeconómico en una población infantil de Caracas. Arch Venez Puer Ped. 2011; 74(2):002-009. Disponible en: http://ve.scielo.org/scielo.php?script=sci_arttext\&pid=S0004-06492011000200002\&lng=es

23. Gil-Gil Beatriz E, Melgar-Quiñonez H, Álvarez-Uribe M, Estrada-Restrepo A. Diferencias en el gasto alimentario según características socioeconómicas y de seguridad alimentaria y nutricional en hogares de Medellín. Perspect Nut Hum. 2017; 19(1):15-25. https://doi.org/10.17533/udea.penh.v19n1a02

24. Ibarra J, Hernández C, Ventura-Vall-Llovera C. Hábitos alimentarios y rendimiento académico en escolares adolescentes de Chile. Rev Esp Nutr Hum Diet. 2019;23(4):292-301. https://dx.doi.org/10.14306/renhyd.23.4.804

25. García MC. Alimentación y rendimiento escolar en adolescentes. Pasaj Cienc. 2011;(14): 99-104. Disponible en: https://www.pasajealaciencia.es/2011/pdf/18-garciamorales.pdf

26. Cabezas-Zábala C, Hernández-Torres B, Vargas-Zárate M. Aceites y grasas: efectos en la salud y regulación mundial. Rev Fac Med. 2016;64(4):761-8. http://dx.doi.org/10.15446/revfacmed.v64n4.53684

27. Peralta L, Cerna B. Estado nutricional y el rendimiento académico en los estudiantes universitarios, 2016. YACHAQ. 2019;2(1):62-2. Disponible en: https://revista.uct.edu.pe/index.php/YACHAQ/article/view/83 
28. Landeros RP, Gómez CZ, Rimoldi MJ, et al. Índice de masa corporal y rendimiento académico en estudiantes universitarios. Rev Salud Publica Nutr. 2018;17(4):34-40. Disponible en: https:/www.medigraphic.com/pdfs/revsalpubnut/spn-2018/ spn184d.pdf

29. Zuniga-Jara S, Pizarro-Díaz R, Escudero-López M, Honores-Marí G. Rendimiento Académico Universitario y Conductas Alimentarias. Rev Form Univ. 2018; 11(2), 57-64. http://dx.doi.org/10.4067/S0718-50062018000200057

30. Trigueros R, Aguilar-Parra JM, Cangas AJ, Bermejo R, Ferrandiz C, López-Liria R. Influence of emotional intelligence, motivation and resilience on academic performance and the adoption of healthy lifestyle habits among adolescents. Int $\mathrm{J}$ Environ Res Public Health. 2019;16(16):2810. https://doi.org/10.3390/ijerph16162810

31. Solis ME. Percepción y consumo del desayuno familiar y su relación con el estado nutricional de los adolescentes de colegios públicos de la Ciudad de Arequipa 2016. Arequipa-Perú; Universidad Nacional de San Agustín; 2018, 62 pp. Disponible en: http://repositorio.unsa.edu.pe/bitstream/handle/UNSA/5550/BIMsofemes.pdf?sequence=1\&isAllowed=y

32. Losada A, Leonardelli E, Magliola M. Influencia sociocultural y los trastornos de la conducta alimentaria en adolescentes. Rev Elec Psicol Iztacala. 2015;18(1): 1-37. Disponible en: https://www.medigraphic.com/pdfs/epsicologia/epi-2015/epi151s.pdf

33. Amaya-Hernández A, Alvarez-Rayón G, Ortega-Luyando M, Mancilla-Díaz J. Peer influence in preadolescents and adolescents: A predictor of body dissatisfaction and disordered eating behaviors. Rev Mex Trastor Aliment. 2017;8(1):31-9. https://doi.org/10.1016/j.rmta.2016.12.001

Anexo. Resumen de preguntas del cuestionario de hábitos alimenticios del grupo GRIINSAN

\begin{tabular}{ll}
\hline Preguntas formuladas & Información derivada \\
\hline $\begin{array}{l}\text { Aspecto sociodemográfico } \\
\text { ¿A qué precio compró los alimentos con respecto a la última vez que los adquirió? }\end{array}$ & $\begin{array}{c}\text { Sexo } \\
\text { Gastosto diarios destinados para la alimentación }\end{array}$ \\
¿Quién provee generalmente los alimentos? & Disponibilidad y acceso a los alimentos \\
¿Cuántas personas comen de la misma preparación diariamente? & \\
Consumo de media mañana & \\
Consumo de almuerzo & \\
Consumo de media tarde & Hábitos de consumo (consumo y frecuencia de \\
Consumo de cena & consumo en escala diaria, semanal, quincenal y \\
¿Siente que la alimentación que consume satisface sus necesidades nutricionales? \\
¿En qué momento considera que tiene más hambre? & mensual) \\
¿Es alérgico o intolerante a algún alimento? & \\
¿Prefiere el consumo de alimentos fritos? & \\
Consumo de carnes & \\
Consumo de cereales & \\
Consumo de raíces & \\
Consumo de lácteos & \\
Consumo de frutas & \\
Consumo de verduras & \\
Consumo de leguminosas & \\
Consumo de grasas & \\
Consumo de azucares & \\
Consumo de comidas rápidas & \\
\hline
\end{tabular}

